\title{
URINARY INCONTINENCE
}

\section{PACING BLADDER}

\section{ELECTRICAL ACTIVITY}

The urinary bladder, similar to the heart, is a hollow contractile organ that generates tonic contractions owing to a pacemaker potential. Cardiologists have been able to monitor and modulate this electrical activity for several decades, and now preliminary investigations suggest that this might also be feasible in the urinary bladder.

A total of eight patients undergoing cystoscopy owing to haematuria and/or urinary incontinence had a quadripolar diagnostic electrophysiology catheter introduced through the operating channel of the cystoscope. A moderate level of bladder distention was generated in order to create a flat surface for electrode application. Recording of bladder electrical activity was possible in seven of eight patients; recordings were not possible in one patient owing to equipment failure.

Recordings from the bladder dome both revealed the presence of a wandering baseline, with irregular, spontaneous unidirectional spikes similar in shape to those of smooth muscle action potentials, whereas more consistent spontaneous depolarizations were observed in the trigone. Four patients also underwent bladder pacing, which resulted in smooth muscle depolarization in all patients, and even voiding in one.

These data indicate that spontaneous electrical activity of bladder smooth muscle, similar to that observed in various in vivo and in vitro studies, can also be monitored in human patients. Pending further investigation, such a technique might enable the electrical alterations observed in patients with bladder overactivity to be better understood and could also guide the targeted delivery of agents such as botulinum toxin to the most appropriate areas.

Peter Sidaway

ORIGINAL ARTICLE Kelley, R. S. et al. A pilot study of cardiac electrophysiology catheters to map and pace bladder electrical activity. Neurourol. Urodyn. http://dx.doi.org/10.1002/nau.23087 (2016) 